\title{
Cuestionario para evaluación de la función sexual en mujeres con prolapso genital y/o incontinencia. Validación de la versión española del "Pelvic Organ Prolapse/Urinary Incontinence Sexual Questionnaire (PISQ-12)"
}

\author{
Espuña Pons $\mathrm{M}^{*}$, Puig Clota $\mathrm{M}^{*}$, González Aguilón $\mathrm{M}^{*}$, Zardain $\mathrm{PC}^{* *}$, Rebollo Álvarez P**. \\ *Institut Clinic de Ginecología Obstetricia i Neonatología. Hospital Clinic. Universidad de Barcelona. \\ ${ }^{* *}$ BAP Health Outcomes. Oviedo. Asturias.
}

Actas Urol Esp. 2008; 32(2):211-219

\begin{abstract}
RESUMEN
CUESTIONARIO PARA EVALUACIÓN DE LA FUNCIÓN SEXUAL EN MUJERES CON PROLAPSO GENITAL Y/O INCONTINENCIA. VALIDACIÓN DE LA VERSIÓN ESPAÑOLA DEL "PELVIC ORGAN PROLAPSE/URINARY INCONTINENCE SEXUAL QUESTIONNAIRE (PISQ-12)”

Este artículo resume el trabajo realizado para adaptar culturalmente y validar para su uso en España la versión corta el Pelvic Organ Prolapse/Urinary Incontinence Sexual Questionnaire (PISQ-12), que se convertirá en el primer cuestionario validado en este país específico para la evaluación de la función sexual en mujeres con prolapso genital y/o incontinencia urinaria (IU).

Pacientes y método. Participaron 49 mujeres que consultaron por síntomas de suelo pélvico en una unidad especializada. Las pacientes cumplimentaron: la versión española del cuestionario a examen, el PISQ-12; el cuestionario de evaluación de la IU, ICIQ-UI-SF; el cuestionario de Función Sexual de la Mujer (FSM); y el Cuestionario de Autoevaluación del Control de la Vejiga (CACV). Se calcularon factibilidad, fiabilidad y validez del nuevo cuestionario.

Resultados. Factibilidad: el 99,83\% de la muestra contestó a todos los items; el tiempo medio de cumplimentación fue de 3,5 $(1,5)$ minutos. Fiabilidad: el coeficiente alfa de Cronbach fue de 0,829. Validez: La correlación del PISQ-12 con el FSM fue de 0,71; con el ICIQ-UI-SF de -0,038; con la dimensión "síntomas" del CACV, de -0,30 y con la dimensión "molestias" de -0,40. Mediante análisis factorial se comprueba la existencia en la versión adaptada al español de las mismas 3 dimensiones de la versión original del PISQ-12. Las puntuaciones en el PISQ-12 fueron menores (peores) en mujeres con síntomas y molestias de VH medidas mediante el cuestionario CACV y en mujeres con disfunción sexual medida mediante el FSM.

Conclusiones. El PISQ-12 es un instrumento con las adecuadas características psicométricas para evaluar en España la función sexual en mujeres con problemas de suelo pélvico.
\end{abstract}

Palabras clave: Disfunción del suelo pélvico. Prolapso de órgano pélvico. Disfunción sexual. Incontinencia urinaria.

\section{ABSTRACT}

QUESTIONNAIRE FOR EVALUATION OF SEXUAL FUNCTION IN WOMEN WITH GENITAL PROLAPSE AND/OR INCONTINENCE. VALIDATION OF THE SPANISH VERSION OF "PELVIC ORGAN PROLAPSE/URINARY INCONTINENCE SEXUAL QUESTIONNAIRE (PISQ-12)"

This article summarizes the work done to adapt and to validate the short form of Pelvic Organ Prolapse / Urinary Incontinence Sexual Questionnaire (PISQ-12) for its use in Spain. It will become the first validated questionnaire in this country for the evaluation of the sexual function in women with Pelvic Organ Prolapse and/or Urinary Incontinence.

Patients and method. 49 women who visited a specialized unit with symptoms of pelvic floor were included. Patients filled in the Spanish version of the questionnaire to validate (PISQ-12), the Urinary Incontinence Questionnaire (ICIQ-UI-SF); the Female Sexual Function Questionnaire (FSM) and the Bladder Control Autoevaluation Questionnaire (CACV). Factibility, reliability and validity of the new questionnaire were evaluated.

Results. Factibility: $99.83 \%$ of the sample answered all the items (only one patient did not answer one of the items); average administration time 3.5 (1.5) minutes. Reliability: Cronbach's alpha was 0,829. Validity: PISQ-12 correlation with FSM was 0,71; with ICIQ-UI-SF it was -0,038; with the CACV "symptoms" dimension the correlation was - 0,30 and with the "discomfort" dimension it was $-0,40$.The existence of the same three dimensions of the PISQ-12 original version in the adapted Spanish questionnaire is checked through a factorial analysis. The score in PISQ-12 was worse (lower) in the case of women with Hyperactive Bladder symptoms and discomfort measured with the CACV questionnaire and in women with sexual dysfunction measured with FSM.

PISQ-12 is an instrument with the appropriate psychometric characteristics to evaluate sexual function in women with pelvic floor problems.

Keywords: Pelvic Floor Disorders. Pelvic organ prolapsed. Sexual function. Urinary incontinence. 
T a patología del Suelo Pélvico, incluyendo la Sincontinencia urinaria (IU), incontinencia anal (IA) y el prolapso genital, es muy prevalente, afectando aproximadamente a un tercio de las mujeres adultas ${ }^{1}$. Aunque esta patología no supone un riesgo vital para estas mujeres, sus consecuencias sociales y económicas pueden ser importantes ${ }^{2}$. Sus sintomas pueden causar alteraciones significativas e interferir con las actividades de la vida diaria incluyendo la capacidad de funcionamiento físico, social y sexual ${ }^{3}$. Los sintomas que están relacionados con la actividad sexual, como la IU durante el coito, producen un impacto muy importante en la calidad de vida de las mujeres que los pade$\mathrm{cen}^{4}$. Por consiguiente al evaluar clínicamente a las mujeres con problemas de suelo pélvico, es importante tener en cuenta si son sexualmente activas, si no lo son hay que investigar si la inactividad sexual, puede estar relacionada con su problema de suelo pélvico. Asimismo en la investigación de los resultados de tratamientos para estas patologías, hay que tener en cuenta, que cualquier medida que se proponga para resolver los problemas de suelo pélvico, debería ser evaluable desde el punto de vista de la paciente, en cuanto al impacto en su calidad de vida del tratamiento aplicado y su relación con los cambios en los sintomas urinarios, anorectales y sexuales. Para ello es necesario disponer de cuestionarios adecuadamente validados siguiendo una técnica estandarizada.

El cuestionario Pelvic Organ Prolapse/Urinary Incontinence Sexual Questionnaire (PISQ-31), fue creado por Rogers et al. ${ }^{5}$ y diseñado inicialmente como un instrumento específico, fiable, válido y auto-administrado para evaluar la función sexual en mujeres con prolapso de órganos pélvicos o incontinencia (urinaria o fecal). Posteriormente ${ }^{6}$, el mismo grupo de autores presentó la versión reducida de 12 ítems del mismo cuestionario (PISQ-12). Este cuestionario fue desarrollado a partir del análisis de los datos de 99 de las 182 mujeres entrevistadas para generar la versión original (PISQ-31). Aunque existía una versión en lengua española del cuestionario PISQ-317, ésta había sido desarrollada en EEUU para hispanos residentes en el país, y por lo tanto no es válida para el entorno cultural español. No debemos olvidar que cuando se traduce y valida un cuestionario el entorno sociocultural tiene una gran importancia.
Así, hasta donde llega nuestro conocimiento, en España no existía ningún cuestionario adecuadamente traducido y validado para valorar la función sexual en mujeres con IU y/o prolapso genital, por lo cual el presente estudio tuvo como único objetivo adaptar culturalmente y validar para su uso en España el Pelvic Organ Prolapse/ Urinary Incontinence Sexual Questionnaire-12 (PISQ-12), cuestionario específico de evaluación de la función sexual en mujeres con prolapso genital y/o incontinencia urinaria.

\section{SUJETOS Y MÉTODO}

El estudio de adaptación cultural y validación del cuestionario PISQ-12 se llevó a cabo mediante cuatro fases:

Fase 1. Traducción: traducción al español las 12 cuestiones de que consta la versión original norteamericana del cuestionario a fin de obtener una versión semántica y conceptualmente lo más parecida posible a la original. Para ello intervinieron dos traductores profesionales bilingües, nativos de habla española e inglesa. Cada traductor realizó una traducción independiente del cuestionario del inglés al español. El equipo investigador revisó las dos traducciones para identificar cuestiones que pudiesen plantear problemas semánticos o conceptuales y poder resolverlos a la hora de consensuar una versión en español.

Fase 2. Retrotraducción: las 12 preguntas traducidas del inglés al español fueron de nuevo traducidas al inglés por otro traductor bilingüe nativo de habla inglesa. Posteriormente se realizó una reunión de consenso en la que participó el equipo investigador y los traductores implicados en la traducción y retrotraducción generándose la versión española definitiva del cuestionario lista para ser probada en pacientes.

Fase 3. Panel de pacientes y de expertos: el objetivo de esta etapa fue verificar que la población española entiende e interpreta lo mismo al leer los items traducidos, lo que sirve para comprobar la equivalencia conceptual entre la versión norteamericana y española del cuestionario. La versión del cuestionario generada en la etapa 2 fue probada en diez mujeres con incontinencia urinaria y prolapso genital y revisada por tres médicos de una unidad uroginecológica. El cuestionario revisado de acuerdo a los comentarios que aportaron pacientes y expertos generó la versión definitiva española del PISQ-12 (Fig. 1). 


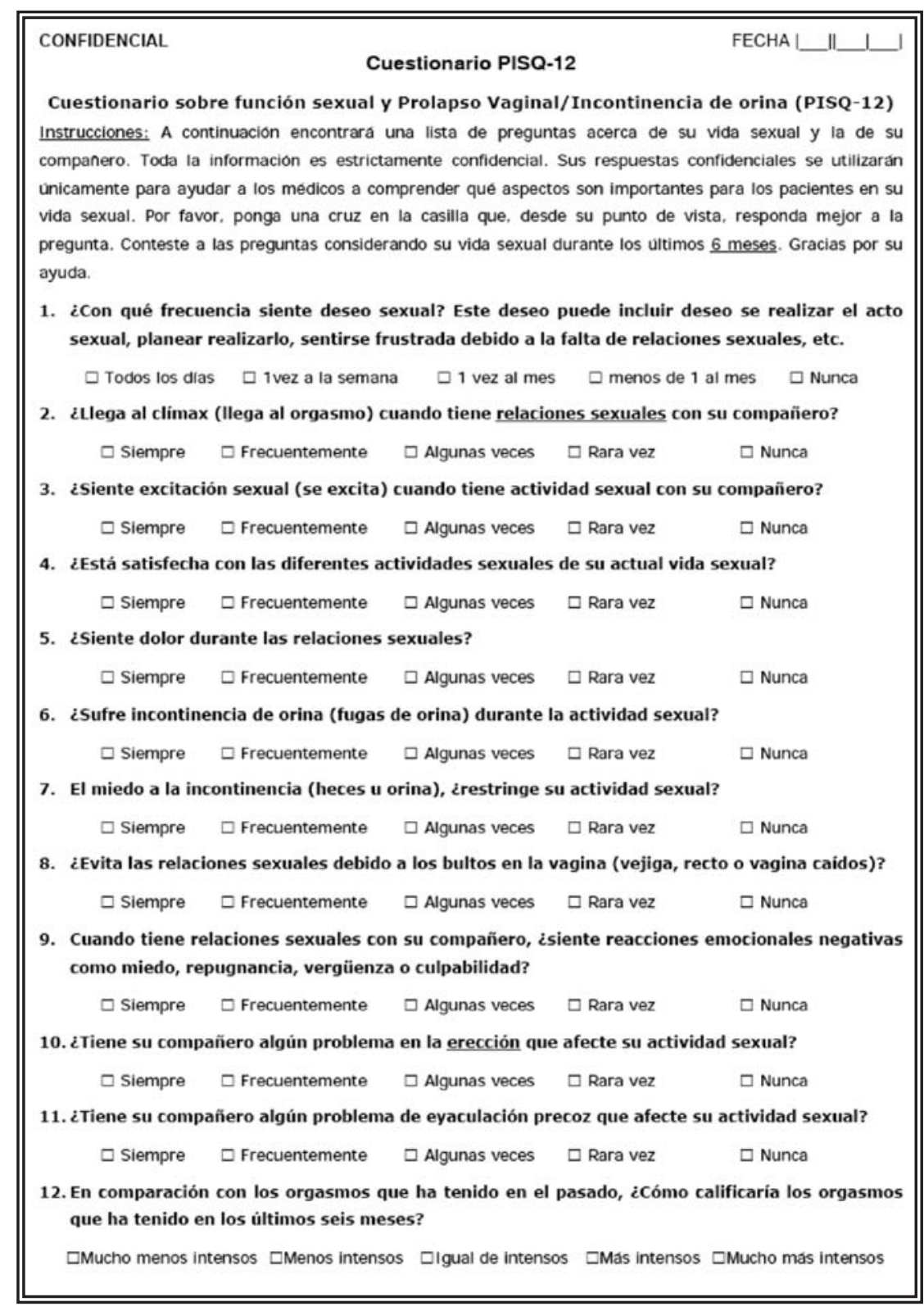

FIGURA 1.

Fase 4. Evaluación de las propiedades psicométricas de la versión española del cuestionario PISQ12: para realizar la validación psicométrica se propuso la participación en el estudio a 100 mujeres que acudieron consecutivamente a la Unidad de Uroginecología del ICGON del Hospital Clínic de Barcelona. Estas mujeres cumplian con los criterios de inclusión de tener prolapso genital o síntomas de incontinencia urinaria $\mathrm{y} / \mathrm{o}$ vejiga hiperactiva, además del de haber mantenido algún tipo de actividad sexual durante los últimos 6 meses. De estas mujeres aceptaron participar un total de 49 .
Todas las mujeres incluidas en el estudio siguieron el protocolo diagnóstico habitual de la unidad consistente en historia uroginecológica y exploración fisica para evaluar defectos del suelo pélvico. Además todas fueron directamente interrogadas acerca de sus sintomas urinarios siguiendo un cuestionario que forma parte de la historia clínica empleada en la citada Unidad. En una hoja de datos estandarizada se recogieron los siguientes datos de cada paciente: datos demográficos (edad, paridad, menopausia, tratamiento hormonal sustitutivo), antecedentes (cirugía correctora de IU, cirugía vaginal, histerectomía); datos de exploración física (talla y peso para calcular el IMC, signos de atrofia vaginal, hipermovilidad uretral, IUE demostrada, cistocele > I grado, rectocele> I grado, prolapso uterino > I grado). En la misma visita las pacientes contestaron junto con el cuestionario a examen, PISQ-12 (Fig. 1), los siuientes cuestionarios: a) versión española del "Internacional Consultation on Incontinence Questionnaire-Urinary Incontinence Short Form" (ICIQ-UI-SF) previamente validada en nuestro país. El ICIQ-UI SF es un cuestionario que consta de 3 items ("Frecuencia", "Cantidad" y "Afectación"), más otros 8 que no forman parte de la puntuación del cuestionario, y cuya finalidad es descriptiva y orientadora sobre el tipo de incontinencia de orina. La puntuación total, resulta de la suma de los tres primeros ítems y oscila entre 0 y 21 puntos $^{8}$. b) Cuestionario de Función Sexual de la Mujer (FSM), diseñado y validado en nuestro país y que consta de 14 items ${ }^{9}$. Para este estudio se tuvo en cuenta la puntuación global del cuestionario y las respuestas a los items pertenecientes a los dominios evaluadores de actividad sexual (DEAS), que se estimó oscilaban entre trastorno 
severo (puntuaciones $\leq 12$ ), trastorno moderado (de 13 a 27) y sin trastorno (de 28 a 60 puntos). Y c) Cuestionario de Autoevaluación del Control de la Vejiga $(\mathrm{CACV})$, también previamente validado en nuestro país; consta de 8 ítems divididos en dos escalas, sintomas y molestias, y ha demostrado adecuadas propiedades psicométricas para ser empleado en la práctica clínica como instrumento de cribado de $\mathrm{VH}$ (puntuaciones $\geq 6$ en cada escala) ${ }^{10}$.

El cálculo de la puntuación del cuestionario PISQ-12, se realizo mediante el sumatorio de las puntuaciones de cada item, (de $0=$ siempre a $4=$ nunca), invirtiéndose esta puntuación para los items 1, 2, 3, y 4.

El método de recogida de datos fue la autocumplimentación de los cuestionarios mediante entrega controlada. Las respuestas a los cuestionarios fueron codificadas y analizadas aplicando reglas de consistencia y rangos que permitiesen detectar inconsistencia y/o errores en la recogida y tabulación de los datos. A continuación se realizaron los análisis estadísticos clásicos de factibilidad, validez y fiabilidad.

\section{Análisis estadístico}

Se ha utilizado el software estadístico SPSS para Windows (versión 14.0) en el desarrollo de una base de datos óptima para los intereses del estudio y para el análisis de los mismos.

Para describir la muestra de acuerdo a las variables personales y clínicas recogidas, se utilizaron las frecuencias absolutas y relativas para las variables cualitativas y la media con su desviación típica para las variables cuantitativas.

Factibilidad: analizada en función de la tasa de respuesta, el tiempo medio de cumplimentación, y la respuesta a los ítems del cuestionario. Para evaluar la respuesta a los ítems se utilizó el tanto por ciento de pacientes que no respondió a cada uno de los mismos.

Fiabilidad: se evaluó la consistencia interna mediante el cálculo del coeficiente alfa de Cronbach.

Validez: la validez de constructo se evaluó analizando los coeficientes de correlación ítemtotal para cada uno de los items del cuestionario a examen y mediante análisis factorial, para comprobar que la versión española del cuestionario tenía la misma estructura factorial que la versión original norteamericana. Se realizó un análisis factorial confirmatorio por el método de Componentes Principales (previa comprobación de la medida de adecuación muestral de Kaiser-Meyer-Olkin y la prueba de esfericidad de Bartlett) con rotación Varimax. La validez de criterio se analizó mediante el cálculo de los coeficientes de correlación de Pearson o Spearman entre las puntuaciones del cuestionario PISQ-12 y las puntuaciones de los otros cuestionarios empleados en el estudio (ICIQUI-SF, CACV y FSM). La validez de concepto se evaluó aplicando la prueba de la T de Student para comparar las medias en la puntuación en el PISQ12 entre subgrupos de la muestra: con y $\sin \mathrm{VH}$ evaluada mediante el cuestionario CACV, y con disfunción sexual moderada versus severa, evaluada mediante el FSM.

\section{RESULTADOS}

Un total de 100 pacientes que reunian criterios de inclusión fueron invitadas a participar en el estudio, de ellas 51 mujeres no quisieron participar, porque consideraron que el cuestionario sobre su función sexual no tenía relación con el problema por el que consultaban. Se estudió a 49 mujeres con una edad media de 47,9 años (D.E.=9,8), un IMC medio de $26,9(5,5)$ y una paridad media de 2,2 hijos (1). La descripción de la muestra respecto a las variables clínicas recogidas en el estudio se presenta en la Tabla 1.

Tabla 1. Descriptivos estadísticos de la muestra

\begin{tabular}{lc}
\hline & N (\%) \\
\hline Media de edad & 47,9 \\
Paridad media & 2,23 \\
Menopausia & $12(25 \%)$ \\
Tratamiento hormonal sustitutivo & $1(2 \%)$ \\
\hline
\end{tabular}

\section{Exploración física}

Signos de atrofia vaginal

$3(6,1 \%)$

Hipermovilidad uretral

$14(28,6 \%)$

IUE demostrada

$7(14,3 \%)$

Cistocele (igual o mayor II grado)

$14(28,6 \%)$

Rectocele (igual o mayor II grado)

$4(8,2 \%)$

Prolapso uterino (igual o mayor II grado) $4(8,2 \%)$

\section{Antecedentes}

Cirugía corrección IU

Cirugia vaginal

$1(2 \%)$

Histerectomía

$3(6,1 \%)$ 
Las puntuaciones medias de las pacientes estudiadas en los cuestionarios CACV, ICIQ-UI, FSM y PISQ-12 se presentan en la Tabla 2.

Tabla 2. Resultados de las puntuaciones medias en los cuestionarios de sintomas de vejiga hiperactiva (CACV), de incontinencia urinaria ( ICIQ-IU-SF) y del de función sexual (PISQ-12), de las pacientes estudiadas

\begin{tabular}{lcccc}
\hline & $\begin{array}{c}\text { CACV* } \\
\text { Sintomas }\end{array}$ & $\begin{array}{c}\text { CACV } \\
\text { Molestias }\end{array}$ & $\begin{array}{c}\text { ICIQ-UI- } \\
\text { SF** }\end{array}$ & $\begin{array}{c}\text { PISQ- } \\
\mathbf{1 2 * * *}\end{array}$ \\
\hline Media & 6,074 & 6,741 & 12,571 & 36,875 \\
D.E. & 2,464 & 3,312 & 4,932 & 7,808 \\
\hline
\end{tabular}

*Cuestionario de Autoevaluación del Control de la Vejiga **International Consultation on Incontinence QuestionnaireUrinary Incontinence Short Form

***Pelvic Organ Prolapse/Urinary Incontinence Sexual Questionnaire-12

Factibilidad: La tasa de respuesta fue del 50\%, en cambio la factibilidad del cuestionario PISQ12 fue prácticamente del $100 \%$, ya que solamente una persona no contestó uno de los 12 items. El tiempo medio de cumplimentación fue de 3,5 $(1,5)$ minutos.

Validez de constructo: Los coeficientes de correlación item-total de cada uno de los ítems del cuestionario PISQ-12 oscilaron entre 0,22 y 0,76 . En el análisis factorial confirmatorio, mediante la extracción de 3 componentes, queda explicado el $66,45 \%$ de la varianza total y se replica la estructura factorial de tres dimensiones de la versión original (Tabla 3).

Fiabilidad: para el cuestionario completo el alfa de Cronbach fue de 0,83. Calculada la fiabilidad de cada una de las dimensiones del cuestionario resultantes del análisis factorial se obtiene un $\alpha=0,86$ para la primera dimensión, $\alpha=0,75$ para la segunda y $\alpha=0,54$ para la tercera.

Validez de criterio: se empleó el coeficiente de correlación de Pearson dado que, aplicada la prueba de Kolmogorov-Smirnov se comprueba que todas las variables tienen una distribución normal y el tamaño y características de la muestra asi lo permiten. Los coeficientes de correlación entre el cuestionario a examen y el resto de cuestionarios del estudio fueron bajas para el ICIQ-UI-SF ( $\mathrm{r}=-0,038 ; \mathrm{p}=0,847)$ y el CACV-síntomas $(r=-0,299 ; p=0,130)$, moderada para el CACV-molestias $(\mathrm{r}=-0,396 ; \mathrm{p}=0,041)$ y moderadaalta para el FSM $(r=0,71 ; p<0,001)$ y el FSM-DS $(\mathrm{r}=0,504 ; \mathrm{p}<0,001)$.

Validez de concepto: Las mujeres con VH de acuerdo a la puntuación en ambas dimensiones del cuestionario CACV (síntomas y molestias) expresaron puntuaciones inferiores (peores) en el cuestionario a examen (Tabla 4). En cuanto al FSM, la puntuación media de la muestra en este

Tabla 3. Resultados del análisis factorial

\begin{tabular}{|c|c|c|c|c|c|c|c|c|c|}
\hline \multirow[t]{2}{*}{ Componente } & \multirow{2}{*}{$\begin{array}{c}\begin{array}{l}\text { Autovalores } \\
\text { iniciales }\end{array} \\
\text { Total }\end{array}$} & \multicolumn{3}{|c|}{$\begin{array}{l}\text { Sumas de saturaciones al } \\
\text { las cuadrado de la extracción }\end{array}$} & \multicolumn{4}{|c|}{$\begin{array}{l}\text { Suma de las saturaciones al cuadrado } \\
\text { de la rotación }\end{array}$} & \multirow[b]{2}{*}{$\begin{array}{c}\% \\
\text { acumulado }\end{array}$} \\
\hline & & $\begin{array}{c}\% \text { de la } \\
\text { varianza }\end{array}$ & $\%$ acumulado & Total & $\begin{array}{c}\% \text { de la } \\
\text { varianza }\end{array}$ & $\%$ acumulado & Total & $\begin{array}{l}\% \text { de la } \\
\text { varianza }\end{array}$ & \\
\hline 1 & 4,483 & 37,356 & 37,356 & 4,483 & 37,356 & 37,356 & 3,645 & 30,378 & 30,378 \\
\hline 2 & 1,904 & 15,865 & 53,222 & 1,904 & 15,865 & 53,222 & 2,431 & 20,258 & 50,636 \\
\hline 3 & 1,587 & 13,229 & 66,450 & 1,587 & 13,229 & 66,450 & 1,898 & 15,814 & 66,450 \\
\hline 4 & 1,019 & 8,492 & 74,942 & & & & & & \\
\hline 5 & ,840 & 7,003 & 81,945 & & & & & & \\
\hline 6 &, 555 & 4,624 & 86,570 & & & & & & \\
\hline 7 & ,437 & 3,642 & 90,211 & & & & & & \\
\hline 8 & ,368 & 3,064 & 93,275 & & & & & & \\
\hline 9 & ,279 & 2,323 & 95,598 & & & & & & \\
\hline 10 & ,255 & 2,122 & 97,720 & & & & & & \\
\hline 11 &, 145 & 1,207 & 98,927 & & & & & & \\
\hline 12 &, 129 & 1,073 & 100,000 & & & & & & \\
\hline
\end{tabular}

Método de extracción: Análisis de Componentes principales 
Tabla 4. Diferencias de medias en las puntuaciones en el PISQ-12 en mujeres con síntomas o molestias de VH (puntuación $>6$ ) y sin sintomas o molestias de Vejiga Hiperactiva

Diferencias de medias en las puntuaciones en el PISQ-12 en mujeres con y sin sintomas o molestias de VH T-test- N.C. $95 \%$

\begin{tabular}{llccccc}
\hline & N & Media & D. E. & Diferencia de medias & Sig. (bilateral) \\
\hline PISQ-12 & $\begin{array}{l}\text { Mujeres sin VH } \\
\text { Sintomas } \\
\text { Mujeres con VH }\end{array}$ & 13 & 41,38 & 4,97 & 6,18 &, 03 \\
$\begin{array}{l}\text { Sintomas } \\
\text { PISQ-12 }\end{array}$ & 34 & 35,20 & 8,05 & &, 001 \\
& $\begin{array}{l}\text { Mujeres sin VH } \\
\text { Molestias }\end{array}$ & 10 & 43,6 & 2,72 & 8,5 & \\
$\begin{array}{l}\text { Mujeres con VH } \\
\text { Molestias }\end{array}$ & 37 & 35,105 & 7,25 & & \\
\hline
\end{tabular}

cuestionario fue de 46,56 (D.E.7,542), esta puntuación total nos sirvió para observar que se producía la tendencia correcta (a mayor puntuación en el FSM mayor puntuación en el PISQ-12. En cuanto a la puntuación en los ítems DEAS (dominios evaluadores de actividad sexual), tenemos que 4 personas $(8,2 \%)$ presentaron trastorno sexual severo, y 44 personas $(89,8 \%)$ presentaron trastorno sexual moderado; la media de la muestra en la puntuación en estos ítems fue de 19,02 $(4,380)$. Los resultados de la comparación de las puntuaciones en el PISQ-12 en los sujetos con disfunción sexual moderada y disfunción sexual severa, evaluada mediante el FSM, aparecen en la Figura 2 y van en la dirección esperada, mayor puntuación en el PISQ-12 a medida que aparece menor disfunción sexual.

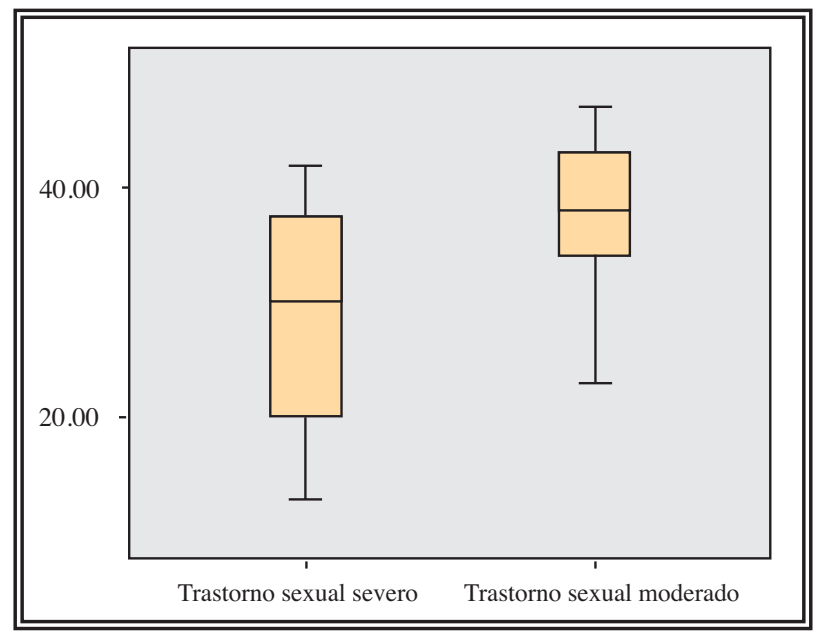

FIGURA 2. Diferencias en las puntuaciones en el PISQ-12* según presenten las pacientes un trastorno de la función sexual moderado (de 13 a 27) o severo ( $\leq 12)$ de acuerdo con la puntuación en el FSM-DS**9.

\section{DISCUSIŌN}

Hasta ahora, tanto los clínicos como los investigadores en el ámbito de las disfunciones del suelo pélvico, tenían como objetivo de evaluación, los sintomas urinarios y anorectales y los defectos anatómicos subyacentes, pasando por alto las consecuencias en la función sexual de las mujeres afectas de la patología del suelo pélvico. Las razones que pueden explicar la ausencia de evaluación de la función sexual en las consultas uroginecológicas, son fundamentalmente la falta de tiempo y el conocimiento insuficiente del manejo de las disfunción sexual en la mujer ${ }^{11}$. En nuestro entorno, con frecuencia, existen además barreras culturales que impiden abordar con facilidad este tema en una consulta médica, a la que la paciente acude para buscar solución a otro problema de salud, que ella puede considerar poco relacionado con el motivo de su consulta. Se ha propuesto la utilización de cuestionarios sobre función sexual, pero los denominados genéricos, abordan el tema en profundidad, son a menudo largos y un porcentaje importante de pacientes se niegan a contestarlos porque consideran que no debe ser investigada esta parcela de su vida en una consulta a la que ha acudido por un problema de IU o de prolapso ${ }^{12}$. La posibilidad de disponer de un cuestionario específico, que aborde el tema preguntando directamente sobre el impacto en la función sexual de los síntomas ligados a su patología del suelo pélvico, si además es rápido y fiable, puede facilitar el abordaje de este tema y su cuantificación.

Se presenta el primer cuestionario en España que evalúa adecuadamente la función sexual en 
mujeres con problemas de suelo pélvico, elaborado a partir del cuestionario PISQ-12, mediante estudio de adaptación cultural y validación. La versión española del cuestionario muestra adecuadas factibilidad, fiabilidad y validez, aunque se detectó una elevada tasa de rechazos a responder el cuestionario.

La difícil comparación con un "patrón oro" es uno de los inconvenientes más frecuentes en los estudios de validación de este tipo de escalas, dificultando el análisis de su validez. Creemos que en el presente estudio este inconveniente se ha salvado mediante una comparación múltiple con los cuestionarios que se utilizan mayoritariamente para la evaluación de las disfunciones de suelo pélvico, asumiendo que las correlaciones y las comparaciones de medias entre grupos de pacientes con nuestra versión del PISQ-12 irian en todos los casos en el sentido correcto, como así ha sido. Los problemas de falta de comprensión de las preguntas (por motivos tales como bajo nivel de instrucción o desconocimiento del significado de algún término) fueron solucionados mediante el método de entrega de los cuestionarios, puesto que la entrega controlada no interfiere en la auto-cumplimentación de los mismos y sin embargo pone a disposición del sujeto a una persona capacitada para responder sus dudas.

Una posible limitación del estudio es el pequeño tamaño muestral utilizado: sólo aceptaron 49 de 100 pacientes invitadas a participar. Aunque en principio pudiera parecer una muy baja tasa de participación, no lo es tanto si tenemos en cuenta la índole de las preguntas y las reticencias a responder o la vergüenza que es habitual que provoquen. En estudios precedentes, como los de validación del cuestionario PISQ-31 $1^{5}$, el SHF- $36^{13}$ o el $\mathrm{FSM}^{9}$, otros autores confirman el alto porcentaje de la muestra que rehúsa contestar las preguntas por referirse a cuestiones de deseo y actividad sexual. Esto se ha intentado corregir mediante cuestionarios más breves, como el PISQ-12 o el SHF-12, aunque en el campo de las disfunciones de suelo pélvico los cuestionarios genéricos, como el FSM o el citado SHF-12, no son los más adecuados.

A pesar de la baja tasa de participación, de las 49 mujeres que fueron incluidas en el estudio prácticamente del 100\% cumplimentaron todos los items del cuestionario, comprobándose que la factibilidad del PISQ-12 versión española fue similar a la del cuestionario FSM, a la del CACV y al a la del ICIQ-UI, donde todas las personas que los contestaron respondieron a todos los items. El tiempo medio de cumplimentación fue de 3,5 minutos, igualando, por ejemplo, a la factibilidad del cuestionario CACV.

La fiabilidad del cuestionario es alta. El alfa de Cronbach con los 12 elementos es de 0,83. La fiabilidad de los cuatro componentes pertenecientes a la primera dimensión es de 0,86 , la de los 5 componentes pertenecientes a la dimensión física 0,75 y la de los dos últimos, de 0,54.

La validez de criterio se calculó mediante el análisis de los coeficientes de correlación entre las puntuaciones del cuestionario PISQ-12 y las puntuaciones de los otros cuestionarios empleados en el estudio (ICIQ-UI- SF, CACV y FSM). En resumen, todas las correlaciones, aunque no excesivamente altas, fueron en el sentido correcto: a) correlación PISQ-12/ICIQ-UI-SF: -0,38; $\mathrm{p}=0,847$; se esperaba una correlación negativa con el cuestionario ICIQ-UI-SF (ya que puntuaciones altas en el mismo indican mayor disfunción urinaria y en el caso del PISQ-12 puntuaciones mayores indican menor disfunción sexual), como realmente ocurre, aunque hay que señalar que esta correlación es baja y además, con la muestra de que disponemos, no es estadísticamente significativa. b) con el cuestionario CACV se esperaba igual tipo de correlación que con el ICIQ-UI y se comprueba mediante Pearson que es así, (correlación PISQ-12/CACV-síntomas:-0,30; $\mathrm{p}=0,130$. Correlación PISQ-12/CACV molestias $0,40 ; p=, 041)$ aunque resulta únicamente significativo para el caso de la correlación PISQ12/CACV-molestias donde podríamos considerarla como moderada-baja. Una posible explicación podría basarse en que, desde un punto de vista subjetivo, la percepción individual del síntoma podría realmente interferir con la conducta sexual en mayor medida que el síntoma en sí (por ejemplo, una persona podría indicar en el ítem 4 del CACV que tiene poca pérdida de orina, con lo cual puntuaria bajo en la escala sintomas pero indicar en la escala molestias que le molesta mucho, y por ende afectar en mayor medida a 
su función sexual). Con el cuestionario de Función Sexual de la Mujer (FSM), al que se recurrió como lo más parecido a un "gold standard" del estudio (dado que no existe ningún cuestionario validado en España que mida exactamente lo mismo que el PISQ-12), se esperaba una correlación moderada-alta y positiva y este es el caso donde se obtuvieron mejores resultados $(\mathrm{r}=076$; $\mathrm{p} \leq, 001$ con la puntuación total del cuestionario, y con la puntuación FSM-DS ( $r=0,504 ; p<0,001)$. Especificar que la puntuación total del FSM sirve para comprobar la tendencia, ya que para evaluar disfunción sexual únicamente se han de tener en cuenta los ítems que DEAS (dominios evaluadores de la actividad sexual), cuya media, como se cita en el apartado de resultados, es de 19,020.

En cuanto a la validez de constructo los valores de la correlación elemento-total corregida oscilaron entre 0,22 y 0,76. Aunque las correlaciones ítem-total de dos de estos elementos (items 5 y 11 ) no fue muy alta no se eliminó ninguno porque se comprobó que el aumento en el alfa de Cronbach si se eliminara dicho elemento sería insignificante (de 0,829 a 0,841 en el mejor de estos dos únicos casos que se dieron). El Análisis Factorial exploratorio indica la existencia de 4 agrupaciones de componentes, pero dado que la cuarta de ellas explica muy poca varianza realizamos un análisis factorial confirmatorio, mediante la extracción de 3 componentes, comprobándose que en la versión española aparecen las mismas tres dimensiones que en la versión original del PISQ-12, a saber, Behavioral/Emotive, Physical y Partner-Related, perteneciendo a la primera de ellas los items $1,2,3,4$, a la segunda los ítems $5,6,7,8,9$ y a la tercera los ítems $10,11,12$. La correlación de la primera de estas dimensiones con la puntuación del FSM (items que miden Disfunción sexual) fue superior a 0,7 y significativa, es decir, las personas que puntúan bajo en los ítems del FSM también lo hacen en el PISQ-12, indicando ambas cosas mayor disfunción sexual.

La media de las puntuaciones en el PISQ-12 en las mujeres diagnosticadas de sintomas de $\mathrm{VH}$ fue inferior que la de las que obtuvieron un diagnóstico negativo, obteniéndose una diferencia significativa de 6,185 puntos. En cuanto al diagnóstico de molestias de $\mathrm{VH}$ vemos como las personas sin molestias puntúan más alto en el PISQ12, con una diferencia de medias significativa y aún mayor que en el caso anterior $(8,495)$. Vemos, como era de esperar, que las personas con sintomas o molestias de $\mathrm{VH}$ obtienen una puntuación menor en el PISQ-12, es decir, presentan mayor disfunción sexual. Analizadas estas diferencias de medias para el cuestionario FSM-DS mediante el estudio del diagrama de cajas vemos como las mujeres con un diagnóstico de trastorno severo obtienen puntuaciones más bajas en el PISQ-12, es decir, presentan mayor disfunción sexual, que las que presentan un trastorno moderado (solamente una de las participantes obtuvo el diagnóstico de sin trastorno).

En conclusión, se dispone de una versión adecuadamente adaptada y validada del PISQ12, forma corta del cuestionario Pelvic Organ Prolapse/Urinary Incontinence Sexual Questionnaire (PISQ-31), equivalente a la versión original, que cumple con las propiedades psicométricas de factibilidad, validez y fiabilidad, para ser utilizada en nuestro pais, tanto en la práctica clínica como en la investigación. La versión española del PISQ-12 puede ayudar a identificar las mujeres con patología del suelo pélvico sexualmente activas y a la realización de estudios sobre la función sexual de las mujeres con pareja heterosexual. Así mismo el disponer de un cuestionario corto, puede facilitar el que se utilice para la evaluación de los resultados de los tratamientos, la comparación de las puntuaciones de antes y después de la aplicación del tratamiento, ya sea farmacológico, fisioterapéutico o quirúrgico, nos permite incorporar la visión de la mujer, en un aspecto tan ligado su la calidad de vida, como es la función sexual y con esta perspectiva matizar el concepto de éxito o fracaso del tratamiento. E1 presente estudio pretendia ser una validación preliminar, dado que la validación se ha realizado únicamente en la población para la que fue creado, es decir, en mujeres con disfunción de suelo pélvico. Será necesario confirmar estos resultados en estudios posteriores con una muestra más amplia y un grupo control. 


\section{REFERENCIAS}

1. Mallett VT, Bump RC. The epidemiology of female pelvic floor dysfunction. Curr Opin Obstet Gynecol. 1994;6(4): 308-312.

2. Fultz H, Burgio K, Diokno AC, Kinchen KH, Obenchain R, Bump RC. Burden of stress urinary incontinence for community-dwelling women. Am J Obstet Gynecol. 2003;189(5): 1275-82.

3. Luber KM, Boero S, Choe JY. The demographics of pelvic floor disorders: current observations and future projections. Am J Obstet Gynecol. 2001,184(7):1496-501.

4. Espuna Pons M, Puig Clota M. Coital urinary incontinence: impact on quality of life as measured by the King's Health Questionnaire. Int Urogynecol J Pelvic Floor Dysfunct. 2007 Nov 1 [Epub ahead of print].

5. Rogers RG, Kammerer-Doak D, Villarreal A, Coates K, Qualls C. A new instrument to measure sexual function in women with urinary incontinence or pelvic organ prolapse. Am J Obstet Gynecol. 2001,184(4):552-558.

6. Rogers RG, Coates KW, Kammerer-Doak D, Khalsa S, Qualls C. A short form of the Pelvic Organ Prolapse/Urinary Incontinence Sexual Questionnaire (PISQ-12). Int Urogynecol J Pelvic Floor Dysfunct. 2003;14(3):164-8.

7. Romero AA, Hardart A, Kobak W, Qualls C, Rogers R. Validation of a Spanish version of the Pelvic Organ Prolapse Incontinence Sexual Questionnaire, Obstet Gynecol. 2003; 102(5 Pt 1):1000-1005.

8. Espuña Pons M, Rebollo Alvarez P, Puig Clota M. Validación de la versión española del ICIQ-SF. Un cuestionario para evaluar la incontinencia urinaria. Med Clin (Barc). 2004; 122(8):288-292.
9. Sánchez F, Pérez Conchillo M, Borrás Valls JJ, Gómez Llorens O, Aznar Vicente J, Caballero Martín de Las Mulas A. Diseño y validación del cuestionario de función sexual de la mujer (FSM). Aten Primaria. 2004;34(6):286-294.

10. Espuña Pons M, Puig Clota M, Rebollo Álvarez P. Validación de la versión en español del "Cuestionario de Autoevaluación del Control de la Vejiga (CACV).Un nuevo instrumento para detectar pacientes con disfunción del tracto urinario inferior. Actas Urol Esp. 2006;30(10):10171024.

11. Pauls RN, Segal JL, Silva WA, Kleeman SD, Karram MM. Sexual function in patients presenting to a urogynecology practice. Int Urogynecol J. 2006; 17(6):576-580.

12. Pauls RN, Kleeman SD, Segal JL, Silva WA, Goldenhar LM, Karram MM. Practice patterns of physician members of the American Urogynecologic Society regarding female sexual dysfunction: results of a national survey. Int Urogynecol J Pelvic Floor Dysfunct. 2005; 16(6):460-467.

13. Creti L, Fichten CS, Brender W. Functioning. In: Davis CM, Yaber WH, Bauserman R, Schreer G, Davis SL, editors. Handbook of sexuality-related measures. 2nd. ed. Thousand Oaks (CA): Russell Sage; 1998. p. 261-267.

Correspondencia autora: Dra. M. Espuña Pons Institut Clinic de Ginecología Obstetricia i Neonatología. Hospital Clínic. Universidad de Barcelona.

E-mail autora: 12922mep@comb.es

Información artículo: Original - Sexualidad femenina

Trabajo recibido: noviembre 2007

Trabajo aceptado: diciembre 2007 